\title{
Structural Characterization and Relaxation Processes of the Inner Crystalline Core in Foams Based on Polyethylene/Polypropylene Blends
}

\author{
Rosario Benavente, José Manuel Pereña, Ernesto PÉReZ, \\ Emanuel NEDKOV, ${ }^{*}$ and María Luisa CERRADA ${ }^{\dagger}$ \\ Instituto de Ciencia y Tecnología de Polímeros (CSIC), Juan de la Cierva 3, 28006 Madrid, Spain \\ *Institute of Polymers, Bulgarian Academy of Sciences, 1113 Sofia, Bulgaria
}

(Received April 28, 2003; Accepted August 16, 2003)

\begin{abstract}
Injected foams obtained from blends with different compositions of isotactic polypropylene, iPP, and two different low density polyethylenes, LDPE, were analyzed by X-Ray diffraction, differential scanning calorimetry, and dynamic mechanical thermal analysis. Most of the results on the blends can be simulated as a weighed addition of the two pure components, indicating practical immiscibility. Some features about the crystallization of the iPP component pointed out inhibition and, consequently, a delay in the development of monoclinic crystalline entities.

KEY WORDS Injected Foams / Polyethylene / Polypropylene / Immiscible Blends /

Crystallization /
\end{abstract}

Isotactic polypropylene, iPP, exhibits valuable properties and a very competitive price. Thus, this polymer is one of the most worldwide used commodity plastics. However, its poor low-temperature fracture toughness is a drawback so that it is often blended with elastomeric polymeric materials which improve very effectively the impact resistance of iPP (of course with some reduction of the modulus and strength). These iPP/elastomer systems are generally multiphasic, and optimum impact resistance is achieved by the proper particle size distribution of the dispersed (elastomeric) phase. ${ }^{1}$ Accordingly, blends of iPP with poly(ethyleneco-propylene) (EPR), ethylene-propylene-diene terpolymer rubber (EPDM), poly(styrene-co-butadiene$c o$-styrene) (SBS) and poly(ethylene-co-vinyl acetate) (EVA) have been extensively analyzed and several mechanisms of impact toughening have been proposed. $^{1-5}$

The constituents of a blend and respective content are not unique variables for determining specific properties but the type of processing technique used has significant influence. Therefore, behavior of injection molded structural foams that show a sandwich-like architecture consisting of a cellular core surrounded by a relatively solid integral $\operatorname{skin}^{6-13}$ does not have to be the same possessed by material obtained by compression molding since the morphological details developed might be rather different. Moreover, the formation of a sandwich-like structure depends upon processing conditions and techniques used. The gas-counter process by egression of foamed melt from the core of the molding is an attractive method for manufacturing structural foams. Gas-counter pressure controls skin thickness, density and structure of the cellular core which is affected by the amount of the polymer melt egressed. 6,7,9 Variation of distinct parameters during processing promotes the resulting structure derived within the skin or core. Therefore, two layers are observed in unfoamed skin: an outer amorphous layer and an inner one that is crystalline. Increase in the spherulites size is gradually observed. ${ }^{10}$ Consequently, the crystalline core and spherulites are the largest in the foamed core. This more perfect crystalline structure is probably due to low heat conductivity caused by the gas-phase that keeps the temperature almost constant long enough for the growth of foamed spherulites.

This study comprehensively analyzes viscoelastic relaxation observed in cellular foams injected from binary blends of two LDPE (with different molecular weights) with isotactic PP. Correlation of viscoelastic behavior and structure in the interior of these injected specimens at room temperature was studied for comparison of structural and viscoelastic responses of these blends with other similar compressed molded. Moreover, thermal properties were also examined during first melting, crystallization and second melting processes

\section{EXPERIMENTAL}

\section{Materials}

Two commercially available LDPE different in flowability, Ropoten FB-7-104 (MFI $=5.3$ at $190^{\circ} \mathrm{C}$ at $2.16 \mathrm{Kg})$ and Ropoten $\mathrm{OV}-03-110(\mathrm{MFI}=0.3$ at $190^{\circ} \mathrm{C}$ at $2.16 \mathrm{Kg}$ ), labeled as LDPEa and LDPEb,

${ }^{\dagger}$ To whom correspondence should be addressed (Tel: 34-91-5622900, Fax: 34-91-5644853, E-mail: mlcerrada@ictp.csic.es). 
respectively, were blended with commercial isotactic polypropylene, iPP, (Buplen 6631 with MFI $=2.5$ at $230^{\circ} \mathrm{C}$ at $2.16 \mathrm{Kg}$ ). All of the plain polymers were supplied by Lukoil Neftochim Bourgas (Bulgaria).

Blends different in content of iPP: 25, 50, 75\% by weight, labeled as LDPEa75PP25, LDPEa50PP50, LDPEa25PP75, LDPEb75PP25, LDPEb50PP50, and LDPEb25PP75 respectively, were prepared at $180^{\circ} \mathrm{C}$ as pellets using an extruder-granulator. The different blends were mixed by dry tumbling with azodicarbonamide "Genitron" EPA (blowing agent) at $0.5 \%$ weight, that decomposes at 170 to $210^{\circ} \mathrm{C}$. The foam samples obtained (cylinder diameter, $20 \mathrm{~mm}$ and length, $150 \mathrm{~mm}$ ) were produced by a classical lowpressure process in a mold cavity pre-pressurized with nitrogen, using an on-line injection molding machine KuASY $800 / 250$ fitted with a shut off nozzle to prevent leakage of gas-containing polymer melt before injection. The cylinders were molded through two gates (sprue diameter 4 and $7 \mathrm{~mm}$ ) at melt temperature, $T_{\mathrm{m}}=$ $210^{\circ} \mathrm{C}$; mold temperature, $T_{\mathrm{f}}=200^{\circ} \mathrm{C}$; cooling time, $t=5 \mathrm{~min}$; gas counter pressure, $P_{\mathrm{g}}=1.0 \mathrm{MPa}$. Foamed samples so attained developed an integral structure that consisted of an unfoamed skin and a foamed core.

These cylinders were cut in the middle and specimens from the foamed injected inner core cut parallel to the flow direction were taken and analyzed.

\section{Techniques}

The samples were studied by wide, WAXS, or smallangle X-Ray scattering, SAXS, employing synchrotron radiation (with $\lambda=0.150 \mathrm{~nm}$ ) in the beamline A2 at HASYLAB (Hamburg, Germany). Two linear positionsensitive detectors were used. The SAXS detector, at a distance of $235 \mathrm{~cm}$ from the sample, was calibrated with the different orders of the long spacing of rat-tail cornea $(\mathrm{L}=65 \mathrm{~nm})$. It determined spacings ranged from 5 to $55 \mathrm{~nm}$. The WAXS detector, covering a $2 \theta$ range from about 10 to 30 degrees, was calibrated with the different diffractions of crystalline PET. The data were acquired simultaneously with the two detectors.

An Amplival optical microscope (Carl Zeiss, Jena) was used for analysis of foamed cells within the core of the specimens.

Calorimetric analysis was carried out in a PerkinElmer DSC7 calorimeter, connected to a cooling system and calibrated with different standards. Sample weights ranged from 7 to $9 \mathrm{mg}$. Temperature ranged from $-45^{\circ} \mathrm{C}$ to $200^{\circ} \mathrm{C}$ and the heating/cooling rate was $20^{\circ} \mathrm{C} \mathrm{min}^{-1}$. For crystallinity determinations, values of 290 and $209 \mathrm{~J} \mathrm{~g}^{-1}$ were taken as enthalpy of fusion of perfectly crystalline material in LDPE $^{14}$ and polypropylene, ${ }^{15}$ respectively.
Viscoelastic properties were measured with a Polymer Laboratories MK II dynamic mechanical thermal analyzer in the tensile mode. Real $\left(\mathrm{E}^{\prime}\right)$ and imaginary $\left(E^{\prime \prime}\right)$ components of the complex modulus and loss tangent $(\tan \delta)$ of the samples were determined at 1 , 3,10 , and $30 \mathrm{~Hz}$ at -150 to $150^{\circ} \mathrm{C}$ and heating rate of $1.5^{\circ} \mathrm{C} \mathrm{min}^{-1}$. Apparent activation energy was estimated on loss tangent plots according to an Arrheniustype equation, employing an accuracy of $1{ }^{\circ} \mathrm{C}$ in assignment of loss tangent peaks. Frequency dependence on temperature has been also considered to follow Arrhenius behavior for mechanisms associated to the glass transition though these relaxations are due to cooperative motions. ${ }^{16}$ This approximation can be made without significant error, since the frequencies are low enough to fit linear behavior.

\section{RESULTS AND DISCUSSION}

\section{Structural and Thermal Characterization}

Figure 1 shows cells within the core in LDPEb, PP, and corresponding blends. Shape is quite spherical and spatial distribution is also rather uniform for all of them. However, size and amount are dependent on material analyzed. LDPEb and LDPEb25PP75 exhibit the smallest cells and the largest population. As PP content increased, cells become larger though they are developed in a smaller amount. Similar features are observed when LDPEa is the other component of the blends.

WAXS diffractograms are shown in Figure 2. A common orthorhombic crystal lattice was observed in both LDPE and monoclinic one ( $\alpha$ form) in iPP. WAXS patterns for blends presented a superposition of the different diffractions stemming from both components. Intensity is evidently dependent upon composition in LPDE or iPP, the diffraction profiles being almost identical, inside the experimental error, to those obtained from weighed simulations of the two components in the blend assuming a single mixing law (the molecular weight differences between LDPEa and LDPEb seem not to affect crystallinity within the two set of blends). The crystallinity of any of these two components is not appreciably affected by the presence of the other. This is in contrast to previous results on compressed molded blends of iPP with a metallocene ethylene-1-octene copolymer. ${ }^{17}$ In those blends, an important inhibition of the crystallization capability of the iPP counterpart was found in the blends rich in ethylene-1-octene copolymer. Thus, blends containing $25 \mathrm{wt} \%$ of iPP showed a very reduced iPP crystallinity, in WAXS and DSC curves displayed that, on cooling from the melt, the crystallization exotherm corresponding to iPP was almost absent in the temperature interval at which the ho- 

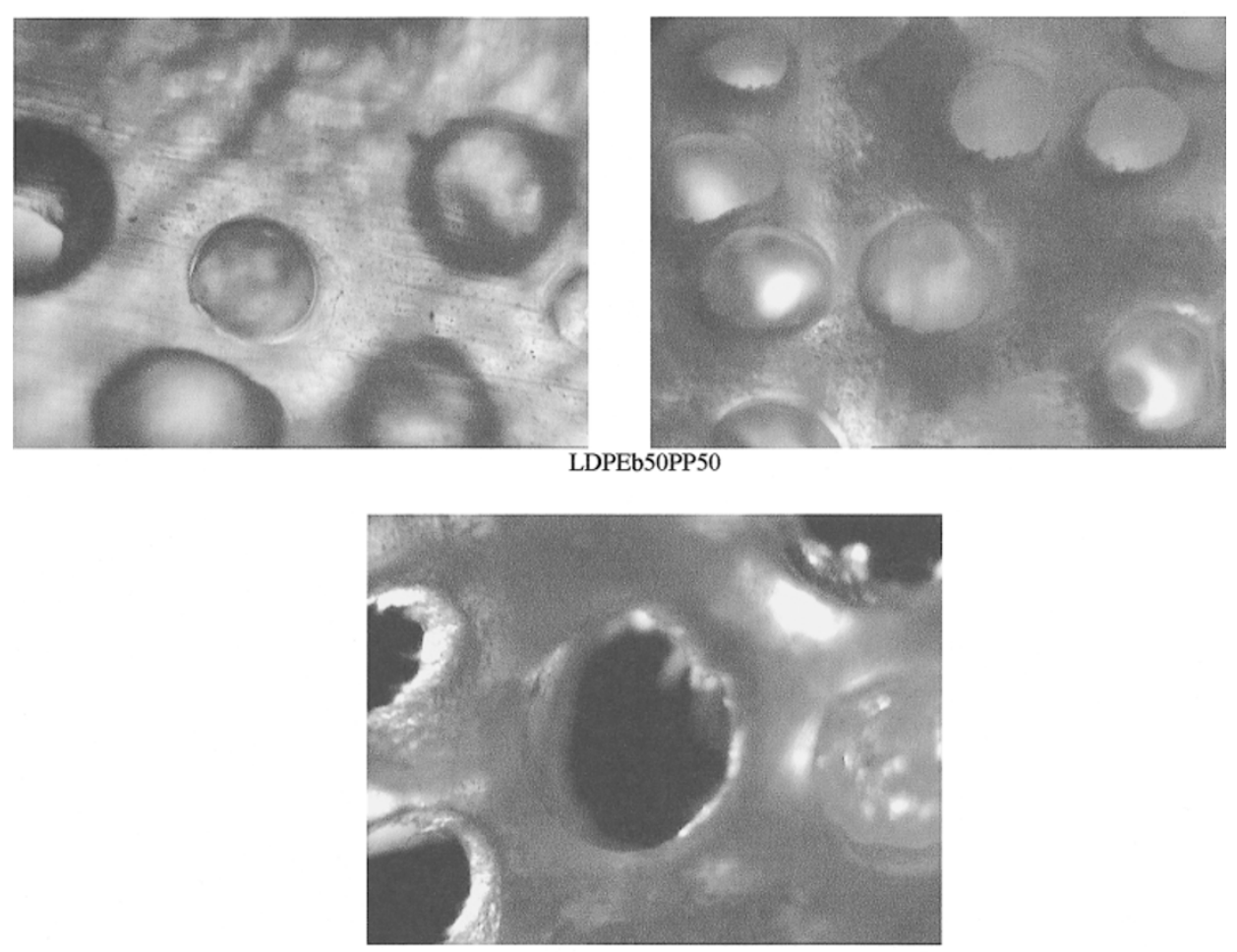

LDPEb25PP75

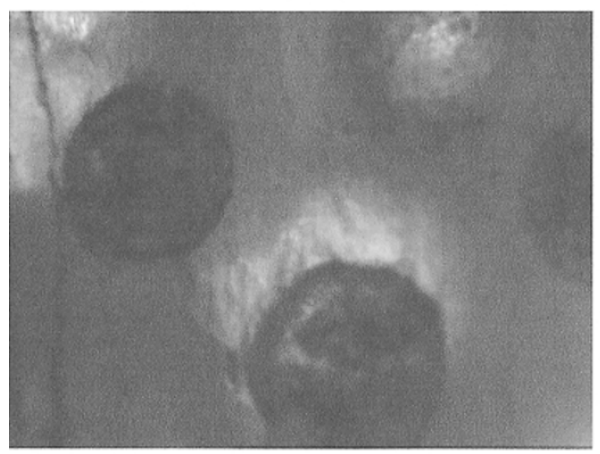

PP

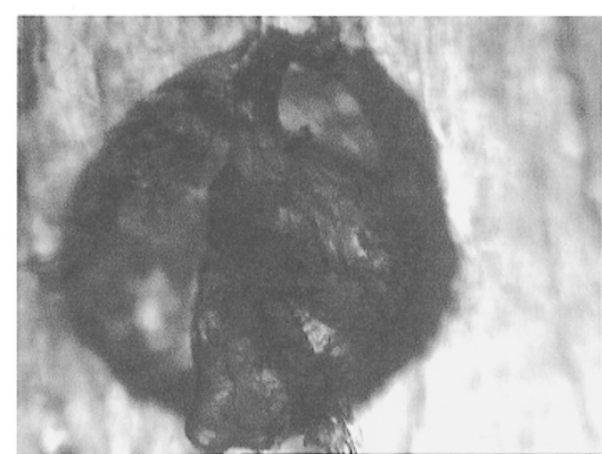

Figure 1. Microscope picture of shape and distribution of foamed cells in LDPEb (upper left), LDPEb75PP25 (upper right), LDPEb50PP50 (middle), LDPEb25PP75 (lower left), and PP (lower right). The total width of each photograph frame represents $1534 \mu \mathrm{m}$.

mopolymer crystallizes, and crystallization of this component took place simultaneously to that of the ethylene copolymer.

To analyze this aspect, a DSC study was conducted on the injected blends. Figure 3 depicts thermal behavior of pure components and blends during the first melting. This first melting represents the structure developed after injection. The two components within blends showed their own melting processes at the same temperatures as in the neat homopolymers, indicating that the two crystalline phases are not cocrystallizable and both types of crystallites remained in the blends, as deduced from the WAXS results. Therefore, the distinct blends show two melting processes related to LDPE, at the low temperature, and iPP fraction. As LDPE content increases the melting endotherm becomes more significant whereas the PP melting peak diminishes. There are other two characteristic regions, one located at about $0{ }^{\circ} \mathrm{C}$ associated to the glass transition of the iPP being visible in all of the blends independently of the composition, as depicted in the insert of Figure 3 for LDPEa blends, and another region at $55-75^{\circ} \mathrm{C}$ identified with an annealing process with recrystallization due to the stay of specimens at room temperature. ${ }^{18}$

Crystallization by cooling from molten state to $-45^{\circ} \mathrm{C}$ at $20^{\circ} \mathrm{C} \mathrm{min}^{-1}$ and subsequent second melting were analyzed in these blends (Figures 4 and 5). In the blends, melting temperatures for LDPE and PP components remained practically constant compared to those found in the neat homopolymers. However, crystallization temperatures, $T_{\mathrm{c}}$, shifted depending on blend composition to higher and lower temperatures for LDPE 


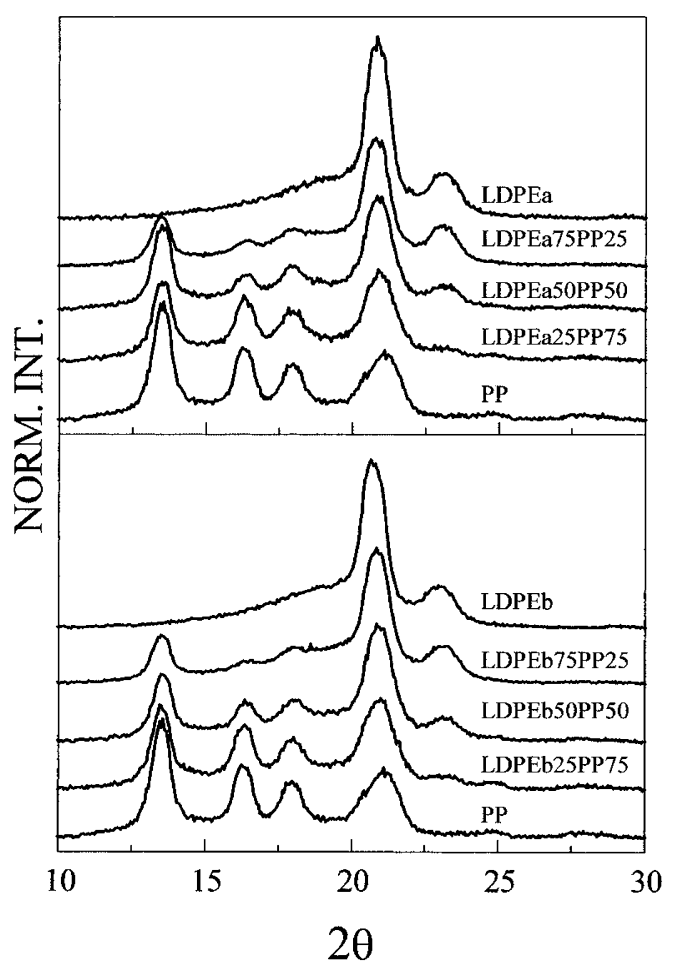

Figure 2. X-Ray diffraction patterns, at room temperature, of different samples. Upper plot for blends of LDPEa and lower plot for blends of LDPEb, with PP at different compositions.

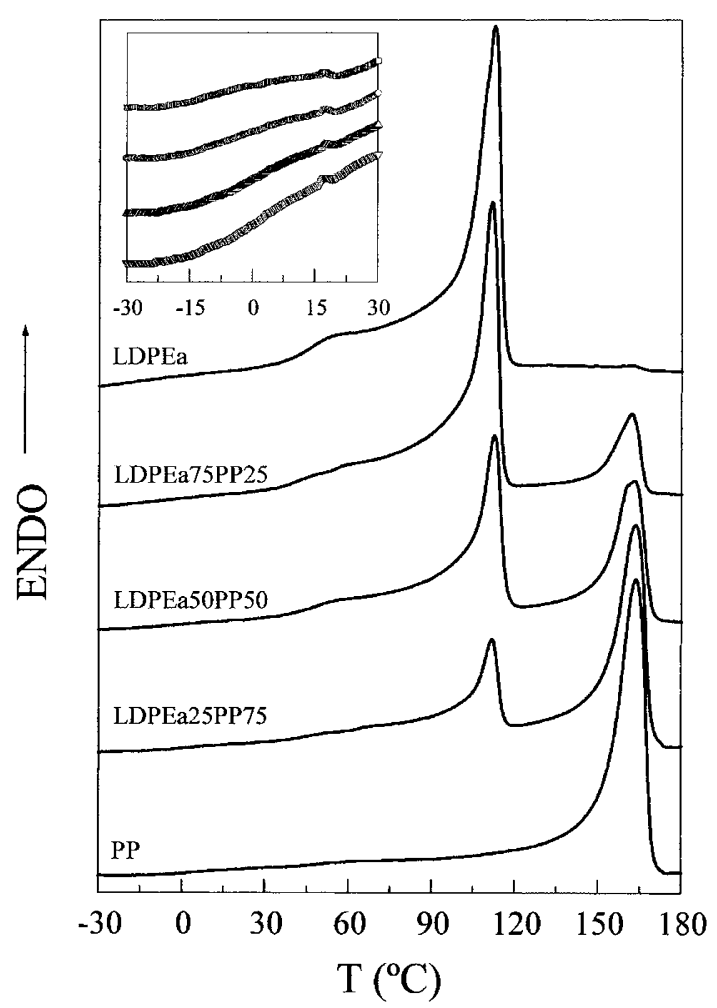

Figure 3. DSC melting curves for plain homopolymers and blends of LDPEa, corresponding to first melting after foam injected processing. Insert: glass transition region of PP (order of the samples as in the main figure, though, evidently, the LDPEa sample is not plotted).

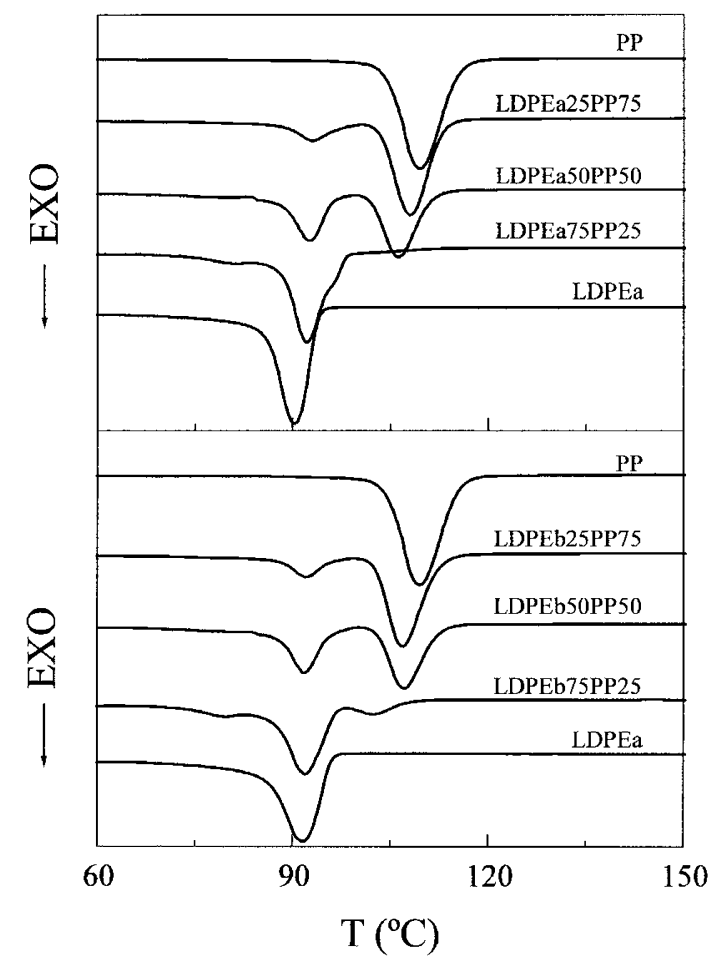

Figure 4. DSC crystallization curves corresponding to LD$\mathrm{PEa} / \mathrm{PP}$ (upper plot) and the LDPEb/PP (lower plot) blends.

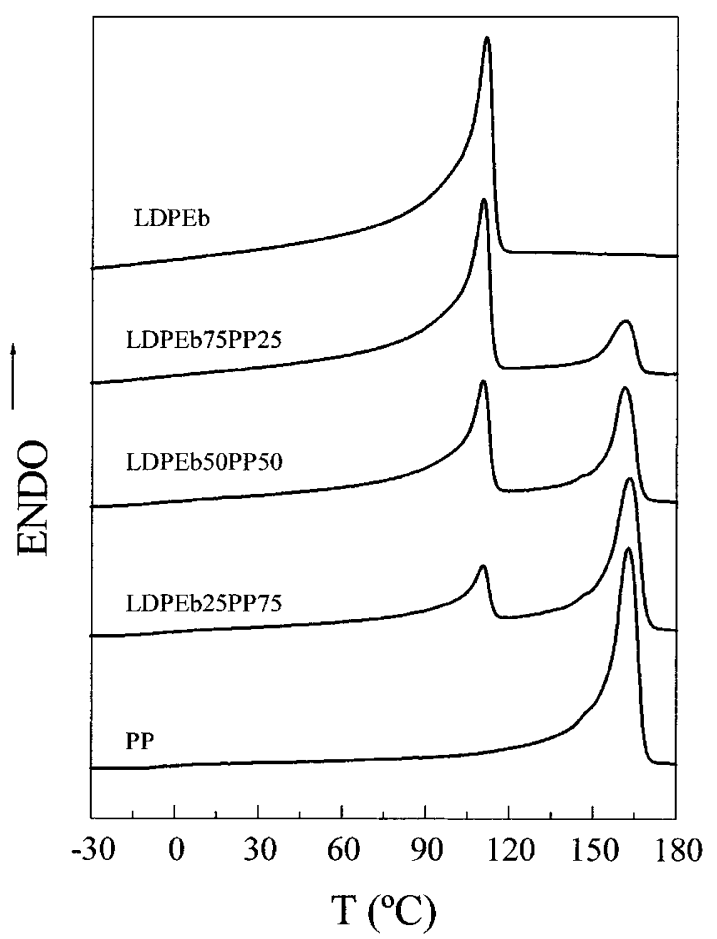

Figure 5. DSC second melting curves corresponding to LD$\mathrm{PEb} / \mathrm{PP}$ blends.

and PP, respectively, as seen in Figure 4 and Table I. Therefore, both $T_{\mathrm{c}}$ 's are getting closer one to the other and they completely merged for the LDPEa75PP25. This $T_{\mathrm{c}}$ displacement was more important as the molecular weight of the LDPE is lowered, as shown in Figure 4 . In the blends with only $25 \%$ of PP, it seems 
Table I. DSC values of the glass transition temperature, melting temperatures for the first and second melting processes and crystallization temperatures of the LDPE and PP components within different blends ${ }^{\mathrm{a}}$

\begin{tabular}{|c|c|c|c|c|c|c|c|c|}
\hline \multirow{2}{*}{ Sample } & \multicolumn{3}{|c|}{ First melting } & \multicolumn{2}{|c|}{ Crystallization } & \multicolumn{3}{|c|}{ Second melting } \\
\hline & $\overline{T_{\mathrm{g}}{ }^{\mathrm{PP}}}$ & $T_{\mathrm{m}}{ }^{\mathrm{LDPE}}$ & $T_{\mathrm{m}}^{\mathrm{PP}}$ & $T_{\mathrm{c}}{ }^{\mathrm{LDPE}}$ & $T_{\mathrm{c}}{ }^{\mathrm{PP}}$ & $\overline{T_{\mathrm{g}}{ }^{\mathrm{PP}}}$ & $T_{\mathrm{m}}{ }^{\mathrm{LDPE}}$ & $T_{\mathrm{m}}{ }^{\mathrm{PP}}$ \\
\hline LDPEa & - & 112.5 & - & 90.5 & - & - & 112.0 & - \\
\hline LDPEa75PP25 & -5.5 & 111.5 & 162.0 & 92.0 & $96.0,80.0$ & -5.5 & 112.0 & 162.0 \\
\hline LDPEa50PP50 & -1.5 & 112.5 & 163.0 & 92.5 & 106.0 & -2.0 & 112.0 & 162.5 \\
\hline LDPEa25PP75 & 4.5 & 112.0 & 163.5 & 93.0 & 108.0 & -2.0 & 112.0 & 162.5 \\
\hline LDPEb & - & 110.5 & - & 91.5 & - & - & 111.5 & - \\
\hline LDPEb75PP25 & -3.0 & 110.5 & 162.5 & 92.0 & $102.5,80.0$ & -3.0 & 110.5 & 163.0 \\
\hline LDPEb50PP50 & -1.5 & 110.5 & 162.5 & 91.5 & 107.0 & -1.5 & 110.5 & 161.0 \\
\hline LDPEb25PP75 & 1.5 & 110.5 & 162.5 & 92.0 & 107.0 & -2.5 & 111.0 & 163.0 \\
\hline PP & 9.0 & - & 163.5 & - & 109.5 & -2.5 & - & 162.5 \\
\hline
\end{tabular}

${ }^{\mathrm{a}}$ Estimated errors: temperatures $\pm 0.5^{\circ} \mathrm{C}$

that a significant amount of PP crystallized below the exotherm corresponding to LDPE, judging from a third peak at around $80^{\circ} \mathrm{C}$. The crystallization of the PP fraction in the blends may thus be significantly inhibited or, better, slowed down. Therefore, it took place at a much lower temperature and an important proportion of PP crystallized simultaneously with (or after) the LDPE component. On the contrary, the crystallite formation in LDPE was slightly accelerated and $T_{\mathrm{c}}$ was moved to slightly higher temperatures. In agreement with this explanation, some authors have interpreted that the iPP may be dissolved in the molten state ${ }^{19}$ of LLDPE. On cooling at constant rate to the temperature of PP crystallization, the content of PP in the LDPE-rich matrix was too low to form nuclei, and hence the crystallization of PP was delayed. Only immediately upon crystallization of LDPE at lower temperature, the concentration of PP in the melt increased and can crystallize. LDPE crystallites could act as nuclei for the crystallization of PP. On the other hand, PP crystallites can also act as a nuclei for the LDPE crystallization and, accordingly, speed up the process. Similar effect on crystallization was found in PP blends toughened with metallocene plastomer. ${ }^{17}$ In those blends for the PP with highest molecular weight, the inhibition lasted during the stay of blends at room temperature, as shown by WAXS. However, the current specimens were at ambient temperature and WAXS patterns were similar to those expected based on a single mixing rule.

The second melting showed again two endothermic melting peaks corresponding to those found in plain homopolymers, as depicted in Figure 5, similarly to the curves attained during the first melting. The endothermic transition observed at $55-75^{\circ} \mathrm{C}$ during the first melting was not now seen because no annealing process at room temperature took place in this second melting.

Figure 6 shows the effects of composition and pro-

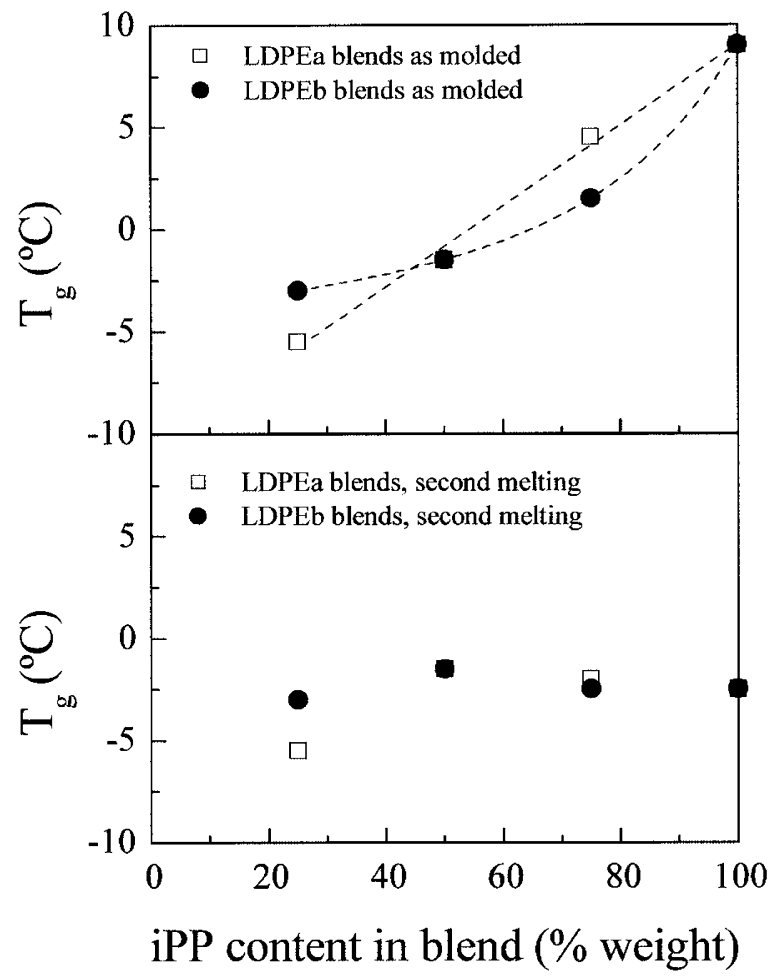

Figure 6. $T_{\mathrm{g}}$ variation of PP component with blend composition during first (open symbols) and second meltings (solid symbols).

cessing conditions on the amorphous regions of iPP component, $T_{\mathrm{g}}$ iPP, within the blends. The thermomechanical history imposed along processing in the injected foams significantly affects the molecular motions within amorphous regions developed in neat iPP and blends with the highest content in this component. Consequently, $T_{\mathrm{g}}$ shifted to higher temperatures due to hindrance in mobility imposed by preferential orientation in the injection direction during foaming. On the other hand, the $T_{\mathrm{g}}{ }^{\mathrm{iPP}}$ values remained constant in those blends where iPP constituent is either in minority or at fifty percent in weight. The dependence of $T_{\mathrm{g}}{ }^{\mathrm{PPP}}$ on iPP composition changed upon LDPE molecular weight. A linear correlation was observed in the blends prepared 


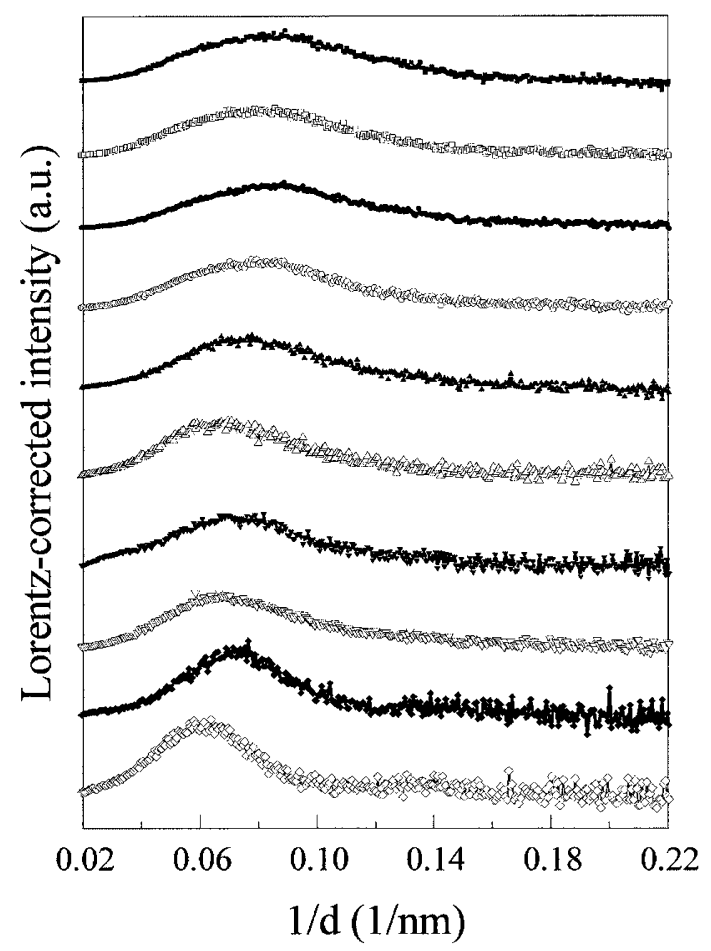

Figure 7. Lorentz-corrected SAXS profiles, at room temperature, for different blends based on LDPEb, as molded or further crystallized at $20^{\circ} \mathrm{C} \mathrm{min}^{-1}$ from the melt. From top to bottom: as molded LDPEb, crystallized LDPEb, as molded LDPEb75PP25, crystallized LDPEb75PP25, as molded LDPEb50PP50, crystallized LDPEb50PP50, as molded LDPEb25PP75, crystallized LD$\mathrm{PEb} 25 \mathrm{PP} 75$, as molded PP, and crystallized PP.

from LDPE with the highest melt flow index whereas an exponential behavior is seen in those blends made from LDPEb. Comparison of $T_{\mathrm{g}}{ }^{\mathrm{PPP}}$ with those found during the second melting process after removing the initial performance by crystallization in the calorimeter showed that $T_{\mathrm{g}}{ }^{\mathrm{iPP}}$ is rather unaffected by blend composition. In iPP/plastomer blends ${ }^{17}$ compressed molded of iPP and blends with a $75 \%$ in weight exhibited a constant $T_{\mathrm{g}}{ }^{\mathrm{PPP}}$ independently of determination from first or second melting process.

Differences due to thermal history were also reflected on long spacing observed because of the existence of crystallites either in the foamed specimens or in those crystallized after their corresponding first melting. Thus, Figure 7 displays the effect of the processing on the inverse of long spacing for the different blends based on LDPEb. At a given sample, initial injected foamed specimen exhibited a long spacing shorter than that observed in specimen crystallized at $20^{\circ} \mathrm{C} \mathrm{min}^{-1}$ probably due to the development of smaller crystallites during injection process because of the high cooling rate. Differences became more important as PP content increases and the largest variation occurred in the plain PP (Figure 7) possibly due to a high sensitivity of $\mathrm{PP}$ with respect to cooling rate.

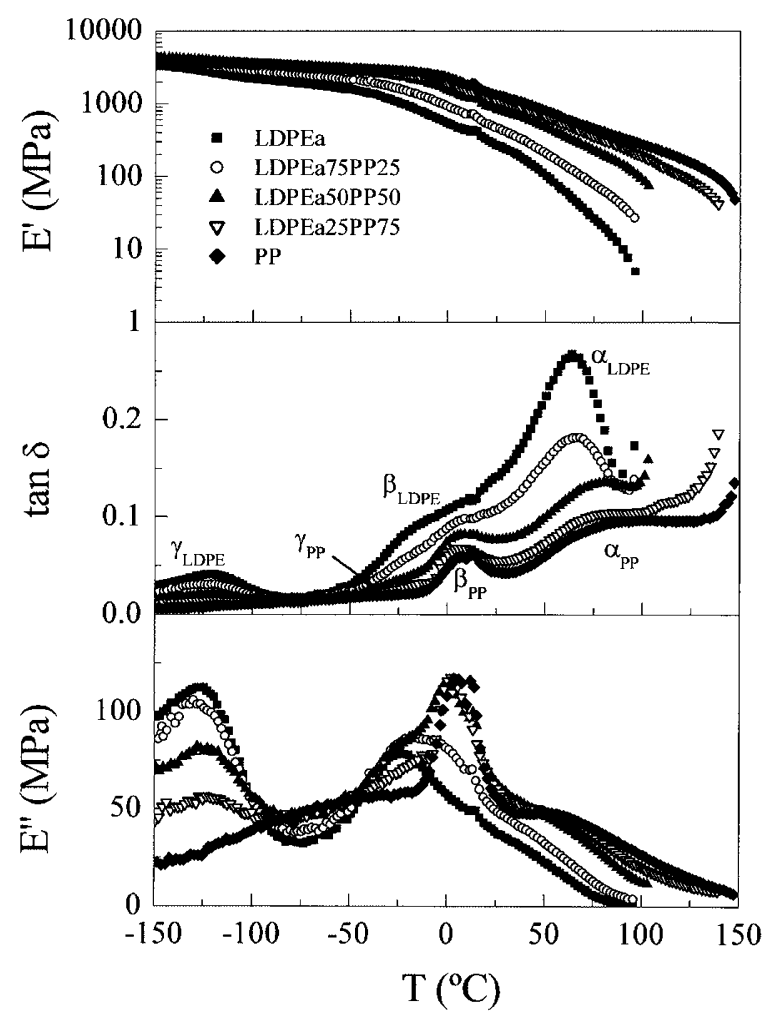

Figure 8. Temperature dependence of real and imaginary components of the complex modulus and loss tangent for LDPEa, PP, and corresponding blends.

\section{Viscoelastic Behavior}

The viscoelastic behavior of the different samples is shown in Figures 8 and 9. Temperature location and apparent activation energy are listed in Table II. LDPE dynamic mechanical spectrum as a function of temperature is well known. ${ }^{20,21}$ Three well-defined relaxation processes were exhibited in LDPEa and LDPEb in the temperature range analyzed (in loss tangent basis): $\gamma_{\mathrm{LDPE}}, \beta_{\mathrm{LDPE}}$, and $\alpha_{\mathrm{LDPE}}$, respectively, in order of increasing temperatures. On the other hand, the plain PP showed three other relaxation mechanisms, labeled as $\gamma_{\mathrm{PP}}, \beta_{\mathrm{PP}}$, and $\alpha_{\mathrm{PP}}$ again in order of increasing temperatures. ${ }^{16,22}$

Figures 8 and 9 show the viscoelastic response of foamed blends for both LDPE. A diminishment of the storage modulus is observed in the blends as LDPE composition increased since the incorporation of the more ductile LDPE decreased significantly the inherent high rigidity of the $\mathrm{PP}$, mainly above its glass transition temperature. Different relaxations were analyzed separately as follows in order of increasing temperatures.

The $\gamma_{\text {LDPE }}$ relaxation in polyethylene was first attributed to crankshaft movement of polymethylenic chains. ${ }^{23}$ Though a lot of work concerning the $\gamma$ relaxation in polyethylene has been done, there remains no clear consensus regarding the details of the underlying motional process. ${ }^{24,25}$ There is, however, a body 
Table II. Relaxation temperatures, in $\tan \delta$ basis at $3 \mathrm{~Hz}$, and apparent activation energies for the different relaxation processes in the two LDPE's and PP homopolymers and the different blends

\begin{tabular}{|c|c|c|c|c|c|c|c|c|c|c|c|c|}
\hline Sample & $T_{\gamma}{ }^{\mathrm{LDPE}}$ & $T_{\gamma}{ }^{\mathrm{PP}}$ & $T_{\beta}{ }^{\mathrm{LDPE}}$ & $T_{\beta}{ }^{\mathrm{PP}}$ & $T_{\alpha}{ }^{\mathrm{LDPE}}$ & $T_{\alpha}{ }^{\mathrm{PP}}$ & $\Delta \mathrm{H}_{\gamma}{ }^{\mathrm{LDPE}}$ & $\Delta \mathrm{H}_{\gamma}{ }^{\mathrm{PP}}$ & $\Delta \mathrm{H}_{\beta}{ }^{\mathrm{LDPE}}$ & $\Delta \mathrm{H}_{\beta}{ }^{\mathrm{PP}}$ & $\Delta \mathrm{H}_{\alpha}{ }^{\mathrm{LDPE}}$ & $\Delta \mathrm{H}_{\alpha}{ }^{\mathrm{PP}}$ \\
\hline LDPEa & -126 & - & -6 & - & 65 & - & 50 & - & 250 & - & 100 & - \\
\hline LDPEa75PP25 & -127 & -52 & -7 & 5 & 63 & 75 & 50 & 100 & 190 & $>400$ & 100 & - \\
\hline LDPEa50PP50 & -129 & -52 & -11 & 7 & 63 & 80 & 50 & 100 & 150 & $>400$ & 150 & 120 \\
\hline LDPEa25PP75 & -130 & -52 & -13 & 9 & - & 85 & 50 & 100 & 160 & $>400$ & - & 120 \\
\hline LDPEb & -121 & - & -4 & - & 60 & - & 50 & - & 250 & - & 100 & $\cdots$ \\
\hline LDPEb75PP25 & -127 & -52 & -6 & 5 & 56 & 80 & 50 & 100 & 200 & $>400$ & 125 & 140 \\
\hline LDPEb50PP50 & -128 & -52 & -8 & 7 & 51 & 80 & 50 & 100 & 200 & $>400$ & 150 & 140 \\
\hline LDPEb25PP75 & -131 & -52 & -10 & 9 & - & 86 & 50 & 100 & 200 & $>400$ & - & 140 \\
\hline $\mathrm{PP}$ & - & -50 & - & 10 & - & 95 & - & 70 & - & $>400$ & - & 280 \\
\hline
\end{tabular}

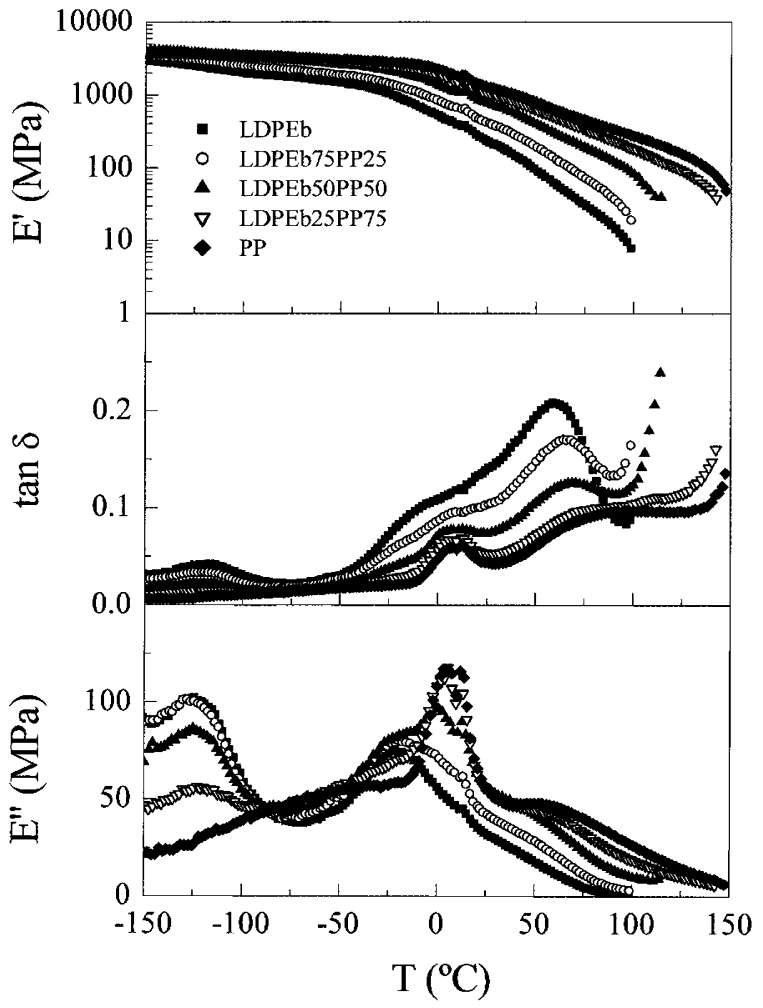

Figure 9. Temperature dependence of storage and loss components of the complex modulus and loss tangent for LDPEb, PP, and corresponding blends.

of opinions which support one or more of the various models for restricted conformational transitions as kink formation, inversion and migration. ${ }^{26-29}$ Molecular dynamics simulation is useful to corroborate the just mentioned nature of these conformational motions. ${ }^{30,31}$ This type of motion requires chains containing sequences of three or more methylenic units. As Table II and Figures 8 and 9 show, $\gamma_{\text {LDPE }}$ relaxation is slightly affected by blend composition and a shift to lower temperature is observed as PP content increases. In addition, the intensity of such process decreases as the PP component is raised in the blend, as expected.

The $\gamma_{\mathrm{PP}}$ is attributed to local motion in the amorphous region in PP. ${ }^{16}$ Its apparent activation energy is quite low and its relaxation time distribution is very broad.

The $\beta_{\text {LDPE }}$ relaxation has been detected at around $-20^{\circ} \mathrm{C}$ in branched polyethylenes, but sometimes appears, though weakly, in linear polyethylene. From study on polyethylenes and copolymers, some authors have concluded that this relaxation results from motion of chain units in the interfacial region ${ }^{32,33}$ whereas some others attributed this process to the glass transition. ${ }^{31,34,35}$ Due to proximity to $\beta_{\text {PP }}$ relaxation, an overlapping of both processes is seen in the blends (Figures 8 and 9).

The $\beta_{\mathrm{PP}}$ process has been identified with the glass transition temperature of amorphous regions in PP. ${ }^{16} \mathrm{In}$ the blends, as seen in Figures 8 and 9, this relaxation appears as an unambiguous peak for LDPEa50PP50, LDPEa25PP75, LDPEb50PP50, and LDPEb25PP75. As PP decreases in the blend, as in LDPEa75PP25 and LDPEb75PP25, the $\beta_{\text {PP }}$ relaxation becomes a shoulder that significantly overlaps with the $\beta_{\mathrm{LDPE}}$ mechanism in its side of high temperatures. Deconvolution of DMTA curves shows that location of this process is dependent upon the blend composition shifting to higher temperatures as iPP content increases. This relaxation appears in these blends and PP homopolymer at higher temperatures than the same process in PP/plastomer blends, ${ }^{36}$ as aforementioned in DSC results. This feature is again ascribed to a motion restriction in PP amorphous regions because of orientation imposed on cylinder core along the processing of foams. The location of this process is independent of the melt flow index of LDPE.

Viscoelastic response was simulated by a single additivity rule (Figure 10) for LDPEa blends. As LDPE increases, the agreement between the experimental and the simulated responses was very good up to the region above the PP glass transition at which a lower intensity than expected was experimentally observed in the interval of $\alpha_{\mathrm{LDPE}}+\alpha_{\mathrm{PP}}$ relaxations (Figure 11). At lowest LDPE content, $\beta_{\text {PP }}$ relaxation associated to PP $T_{\mathrm{g}}$, should appear at higher temperatures if compared to simulated response due to the orientation restrictions in PP. However, it was empirically shifted to 


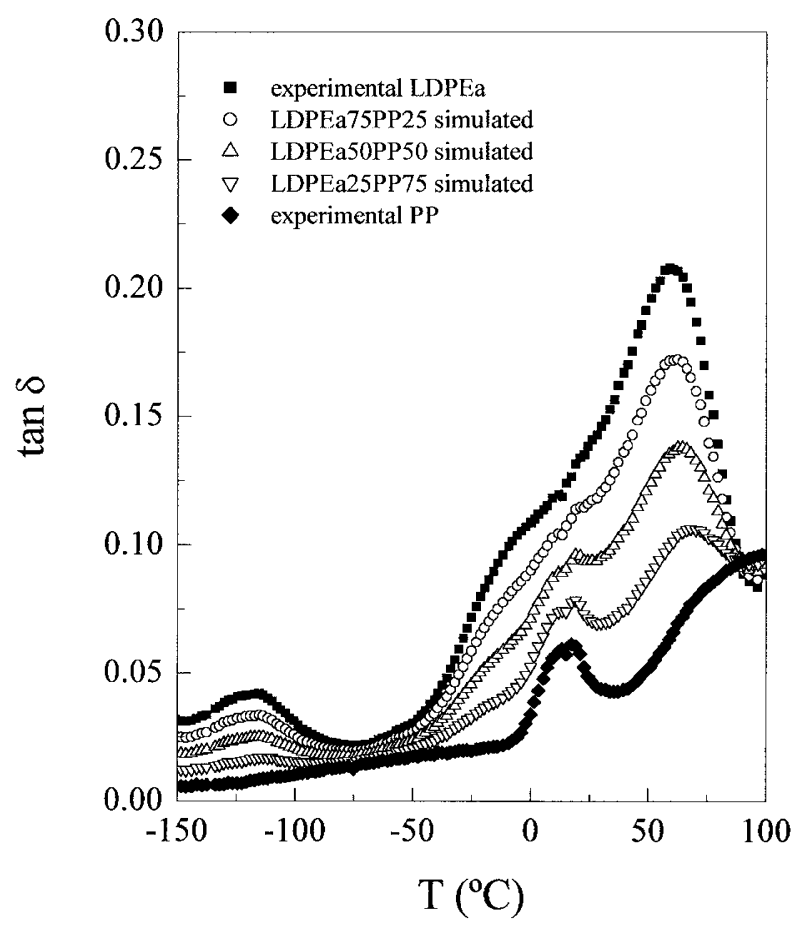

Figure 10. Tan $\delta$ spectra for LDPEa/PP blends simulated from experimental LDPEa and PP $\tan \delta$ responses.

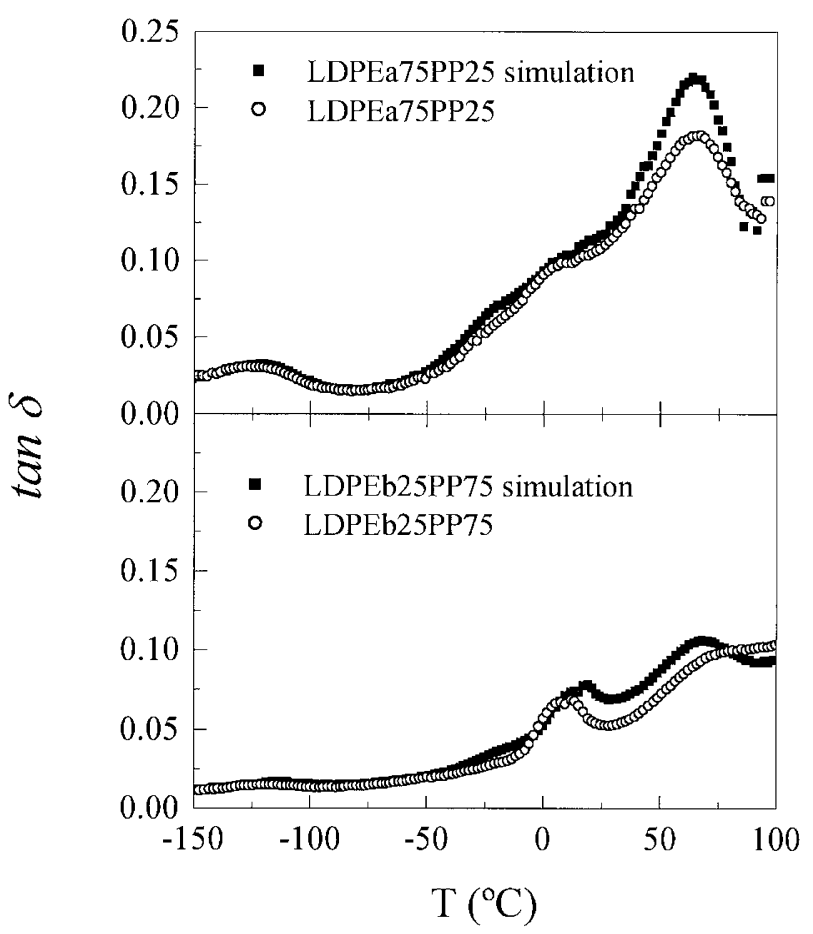

Figure 11. Comparison between simulated and experimental response for blends LDPEa75PP25 (upper plot) and LDPEb25PP75 (lower plot).

lower temperatures. The relaxation time distributions of $\alpha_{\mathrm{LDPE}}+\alpha_{\mathrm{PP}}$ relaxations are experimentally broadened compared with those predicted by the theoretical mixing law.

The $\alpha_{\text {LDPE }}$ relaxation in polyethylene is associated with vibrational and reorientational motion within crys- tallites. ${ }^{16,20}$ This process is overlapped to the $\beta_{\text {LDPE }}$ relaxation in both pure LDPE (Figures 8 and 9). This relaxation appears also merged with the $\alpha_{\mathrm{PP}}$ mechanism in the blends since these two components are not cocrystallizable and both crystalline phases have ability of mobility at those temperatures. This overlapping is apparent by changes in shape, intensity and location with blend composition, as shown in Figures 8 and 9.

The $\alpha_{\mathrm{PP}}$ was clearly observed in iPP and blends with high PP content. It is attributed to movements in the PP crystalline regions. ${ }^{16}$ It has been reported the dependence of its magnitude and relaxation times distribution on the thermal history applied to the polymer. ${ }^{16}$ Its location is also affected by annealing. As the LDPE content increases in the blends under study, a significant merging with the $\alpha_{\text {LDPE }}$ was observed.

\section{CONCLUSIONS}

WAXS structure developed at room temperature by the blends was an overlapping of the diffractions corresponding to both components: LDPE and PP. The foamed and injected-molded homopolymers and their blends exhibited smaller long spacing than themselves prepared by cooling from the melt at $20^{\circ} \mathrm{C} \mathrm{min}^{-1}$, the differences being larger as PP content increases in the blend. Along cooling from the melt, an inhibition or delay in crystallization of the iPP component seemed to take place. Consequently, $T_{\mathrm{c}}$ was moved to a much lower temperature and an important proportion of iPP crystallized simultaneously with, or after, LDPE component did. Moreover, a slight acceleration was found in LDPE constituent within blends and its crystallization was shifted to temperature slightly higher. The thermo-mechanical processing changed appreciably the dependence of PP $T_{\mathrm{g}}$ upon blend composition. An increase of such a $T_{\mathrm{g}}$ was found in the initial injected foams as the PP content was raised, probably due to restrictions in the PP amorphous phase caused by the introduction of orientation along processing, whereas an almost $T_{\mathrm{g}}$ constancy was attained in specimens crystallized at $20^{\circ} \mathrm{C} \mathrm{min}^{-1}$ after melting. Dynamic mechanical measurements showed a diminishment of rigidity of iPP component as the LDPE content increased, mainly above its glass transition. The different relaxation processes observed in the pure components were found in the blends because of the practical immiscibility of these two components for these blends. Supposed that mentioned immiscibility, a simulation of the viscoelastic response with blend composition was made to compare with the empirical behavior. The agreement was quite good for blends with high PP. 
Acknowledgments. The financial support of Comunidad Autónoma de Madrid and Ministerio de Ciencia y Tecnología (Projects 07N/0093/2002 and MAT20012321) is gratefully acknowledged. The synchrotron work (in the polymer line of Hasylab at DESY, Hamburg) was supported by the IHP Programme "Access to Research Infrastructures" of the European Commission (Contract HPRI-CT-1999-00040/2001-00140). We thank the collaboration of the Hasylab personnel, specially Dr. S. Funari, responsible of the polymer beamline.

\section{REFERENCES}

1. B. Z. Jang, D. R. Uhlmann, and J. B. Vander Sande, J. Appl. Polym. Sci., 29, 4377 (1984).

2. Y. Kim, W. J. Cho, and C. S. Ha, Polym. Eng. Sci., 35, 1592 (1995).

3. C. L. Wang, S. J. Wang, and Z. N. Qi, J. Polym. Sci., Part B: Polym. Phys., 34, 193 (1996)

4. A. Van der Wall, R. Mighof, and R. J. Gaymans, Polymer, 40, 6087 (1999).

5. S. H. Jafari and A. K. Gupta, J. Appl. Polym. Sci., 78, 962 (2000).

6. N. Touleshkov, S. Djoumaliisky, and G. Kotzev, Polym. Degrad. Stabil., 24, 327 (1989).

7. G. Kotzev and N. Touleshkov, J. Mater. Sci. Technol., 4, 33 (1996).

8. S. Djoumaliisky and N. Touleshkov, J. Mater. Sci. Technol., 4, 32 (1996).

9. S. Djoumaliisky, N. Touleshkov, and G. Kotzev, Polym.-Plast. Technol. Eng., 36, 257 (1997).

10. S. Djoumaliisky, D. Christova, N. Touleshkov, and E. Nedkov, J. Macramol. Sci., Pure Appl. Chem., A35, 1147 (1998).

11. G. Kotzev, N. Touleshkov, D. Christova, and E. Nedkov, J. Macramol. Sci., Pure Appl. Chem., A35, 1127 (1998).

12. G. Kotzev, N. Touleshkov, D. Christova, and E. Nedkov, J. Cell. Plast., 36, 29 (2000).

13. D. Christova, N. Touleshkov, S. Djoumaliisky, G. Kotzev, and E. Nedkov, Proc. of 9th International Conference on MECHANICS AND TECHNOLOGY OF COMPOSITE MATE-
RIALS, 11-14 (2000).

14. B. Wunderlich, Macromolecular Physics, Academic Press, New York, N.Y., 1980, 3, p. 42.

15. J. R. Isasi, L. Mandelkern, M. J. Galante, and R. G. Alamo, J. Polym. Sci., Part B: Polym. Phys., 37, 323 (1999).

16. N. G. McCrum, B. E. Read, and G. Williams, Anelastic and Dielectric Effects in Polymeric Solids, Chapt. 10, Dover Publications, New York, N.Y., 1991. p. 353.

17. O. Prieto, J. M. Pereña, R. Benavente, M. L. Cerrada, and E. Pérez, Macromol. Chem. Phys., 203, 1844 (2002).

18. A. Alizadeh, L. Richardson, J. Xu, S. McCartney, H. Marand, Y. W. Cheung, and S. Chum, Macromolecules, 32, 6221 (1999).

19. J. Li, R. A. Shanks, R. H. Olley, and G. R. Greenway, Polymer, 42, 7685 (2001).

20. I. M. Ward, Mechanical Properties of Solids Polymers, 2nd. ed, John Wiley and Sons Inc., Chischester UK, 1985.

21. L. E Nielsen and R. F. Landel, Mechanical Properties of Polymers and Composites, 2nd ed, Marcel Dekker, New York, N.Y., 1994.

22. C. Jourdan, J. Y. Cavaille, and J. Perez, J. Polym. Sci., Part B: Polym. Phys., 27, 2361 (1989).

23. F. Schatzki, J. Polym. Sci., 57, 496 (1962).

24. R. G. C. Arridge, Rev. Deform. Behav. Mater., 3, 249 (1981).

25. R. H. Boyd, Polymer, 26, 1123 (1985).

26. R. F. Boyer, Rubb. Chem. Technol., 36, 1303 (1963).

27. R. H. Boyd and R. S. Breitling, Macromolecules, 7, 855 (1974).

28. R. H. Boyd, J. Polym. Sci., Part B: Polym. Phys., 13, 2345 (1975).

29. N. J. Heaton, R. Benavente, E. Pérez, A. Bello, and J. M. Pereña, Polymer, 37, 3791 (1996).

30. R. H. Boyd, R. H. Gee, J. Han, and Y. Jin, J. Chem. Phys., 101, 788 (1994).

31. Y. Jin and R. H. Boyd, J. Chem. Phys., 108, 9912 (1998).

32. R. Popli and L. Mandelkern, Polymer Bull., 9, 260 (1983).

33. R. Popli, M. Glotin, L. Mandelkern, and R. S. Benson, J. Polym. Sci., Part B: Polym. Phys., 22, 407 (1984).

34. R. H. Boyd, Macromolecules, 17, 903 (1984).

35. J. Han, R. H. Gee, and R. H. Boyd, Macromolecules, 27, 7781 (1994).

36. O. Prieto, J. M. Pereña, R. Benavente, E. Pérez, and M. L. Cerrada, J. Polym. Sci., Part B: Polym. Phys., 41, 1878 (2003). 